\title{
Successful Treatment of a Child with Toxic Methemoglobinemia due to Nail Polish Remover Poisoning
}

\author{
SOUMYA PATRA ${ }^{1}$, GURLEEN SIKKA ${ }^{1}$, DEBARSHI BOSE ${ }^{1}$, NEHA PANWAR ${ }^{1}$, AJOY KUMAR \\ KHAOWAS $^{2}$, DEBANJALI SARKAR ${ }^{3}$
}

\begin{abstract}
Children are most susceptible to accidental exposure of common household substances and one of the common household substances is nail polish remover. The chemical N,N-dimethyl-p-toluidine is a chemical commonly found in artificial fingernail solutions. Its oxidation metabolite phenylhydroxylamine is presumably one of the most potent chemical producers of methemoglobin. Fingernail care products account for a significant number of pediatric poisonings in the western world with few fatalities. We are presenting a case of accidental ingestion of nail polish remover with lethal Methemoglobinemia (Serum methemoglobin level-72\%). This patient was treated successfully with injection methylene blue. However, even small amounts can be dangerous to children, so it is important to keep this and all household chemicals in a safe place.
\end{abstract}

Key words: Children; Methemoglobinemia; Methylene blue; Nail polish remover.

\section{Introduction}

Nail polishes and nail polish removers may contain a variety of hydrocarbon-based solvents, while nail adhesives usually contain various acrylic ester monomers; the chemical $N, N$-dimethyl-p-toluidine is a chemical commonly found in artificial fingernail solutions. ${ }^{1}$ Its oxidation metabolite phenyl hydroxylamine is presumably one of the most potent chemical producers of methemoglobin. Accidentally drinking small amounts of acetone/nail polish remover is unlikely to harm in an adult, however even small amounts can be dangerous to children. ${ }^{2}$ Though there are so many reported cases of nail polish remover poisoning in children has been published in western literature ${ }^{1-2}$ but so far no such case was reported in Asia.

\section{Case Report}

A 13 month old male child presented with history of ingestion of small volume of nail polish remover two hours back followed by vomiting and gradual lethargy. After admission the child was kept under observation and was found cyanosed, restless and more lethargic. He had no other significant history. Laboratory

1. Departments of Pediatric, Lady Hardinge Medical College and Kalawati Saran Children's Hospital, New Delhi, India

2. Department of Otolaryngology, N.R.S Medical College, Kolkata

3. Department of Obstetrics \& Gynaecology, MGM Medical College \& L.S.K Hospital, Kishanganj.

Correspondence: Dr Soumya Patr investigations were done and he had all the hematological and biochemical parameters within normal limit. But, he had low oxygen saturation (85$87 \%$ ) done by pulse oxymetry and simultaneously taken arterial blood gas showing $\mathrm{pO}_{2}-157 \mathrm{~mm}$ of $\mathrm{Hg}$ with mildly increased lactate $(1.9 \mathrm{mmol} / \mathrm{lit})$ and the $\mathrm{pH}$ and other parameters were within normal limit. As there was low $\mathrm{SpO} 2$ with increased pO2, we suspected that he must be having methemoglobinemia and investigations like serum methemoglobin level and serum G6PD level were advised. The serum methemoglobin level came out to be $-72 \%$ and G6PD found to be in normal range. As this case was diagnosed as toxic methemoglobinemia, we managed the child with oxygen, intravenous fluids and inj. Methylene blue $(2 \mathrm{mg} / \mathrm{kg})$, single dose. The child responded promptly with the treatment. The cyanosis resolved and child was active and accepted oral feed also. The repeat serum methemoglobin was $2 \%$. The child was discharged after one day with the final diagnosis of toxic methemoglobinemia due to nail polish remover poisoning.

\section{Discussion}

When an infant presents with severe cyanosis which is not associated with respiratory distress, methemoglobinemia should always be suspected. Children affected with methemoglobinemia have a peculiar lavender color. Blood from the heel sticks is 
chocolate-brown and does not become pink when exposed to room air. ${ }^{1}$ In the absence of cyanide level determinations, lethargy, vomiting, seizures, and the lack of normal venous blood hemoglobin desaturation are clues to cyanide toxicity. ${ }^{3}$ Diagnosis can be confirmed by excluding other causes of cyanosis and by spectrophotometric analysis of blood for methemoglobin. When methemoglobin levels reach $70 \%$ or more, the patient will collapse and become comatose and may die. ${ }^{4}$ Therapy with methylene blue results in prompt relief. ${ }^{4}$ Treatment with methylene blue can be complicated by the presence of underlying enzyme deficiencies, including glucose-6-phosphate dehydrogenase deficiency. Health care providers should not confuse the potentially highly toxic acetonitrile-containing cosmetics, particularly falsefingernail removers, with less-toxic acetone-containing fingernail-polish removers. ${ }^{5}$ This potential confusion between acetone and acetonitrile poisoning is compounded by the initial similarity of their early features, including vomiting, lethargy, slurred speech, ataxia, stupor, coma, and respiratory depression. Delayed vomiting, although not typically a major clinical indicator of most cases of cyanide poisoning, may be important in alerting health care providers to acetonitrile toxicity in exposed children. ${ }^{5}$ However, vomiting is common from many causes and is not sufficient by itself to dictate the administration of a cyanide antidote in the absence of other supporting evidence of cyanide toxicity from history and clinical laboratory studies. Sudden death is possible in some nail polish poisoning cases. ${ }^{6}$ The health care provider will measure and monitor the patient's vital signs, including temperature, pulse, breathing rate, and blood pressure. The patient may receive activated charcoal if the patient arrives immediately after swallowing the poison, gastric lavage, oxygen, if needed. ${ }^{6}$ The person survives past 48 hours; the chances for recovery is good. ${ }^{6}$

We wish to draw attention to the potential toxicity of acetone containing nail polish removers and the need for appropriate labeling of product packaging. Since alternative products are available, these products should be removed from the market. Pediatrician should be well versed with this clinical situation.

\section{References}

1. Geller RJ, Ekins BR, Iknoian RC. Cyanide toxicity from acetonitrile- Containing false nail remover. Am J Emerg Med. 1991; 9:268-70.

2. Kurt TL, Day LC, Reed G. Cyanide poisoning from glue-on nail remover. Am J Emerg Med. 1991; 9:271-72.

3. Losek JD, RockAL, Boldt RR. Cyanide poisoning from a cosmetic nail remover. Pediatrics. 1991; 88:337-40.

4. Herranz M, Clerigue N. Poisoning in children. An Sist Sanit Navar. 2003; 26:209-13.

5. Borron SW, Baud FJ. Acute cyanide poisoning: Clinical spectrum, diagnosis, and treatment. Arh Hig Rada Toksikol. 1996; 47:307-22.

6. El-Husseini A, Azarov N. Is threshold for treatment of methemoglobinemia the same for all? A case report and literature review. Am J Emerg Med. 2010; 28:748. 